\title{
Valeurs usuelles des hormones thyroïdiennes dans la population gabonaise : application à la typologie biochimique des hyperthyroïdies
}

\author{
Félix OVONO ABESSOLO ${ }^{1 *}$, Serge BEKALE ${ }^{1}$, Adaku LIAMIDI ${ }^{1}$, Ntyonga PONO ${ }^{2}$ et \\ Edouard NGOU-MILAMA ${ }^{1}$ \\ ${ }^{1}$ Laboratoire de Biochimie, Faculté de Médecine et des Sciences de la Santé, BP 4009 Libreville-Gabon. \\ ${ }^{2}$ Service d'Endocrinologie du Centre Hospitalier de Libreville, BP 2228 Libreville-Gabon. \\ *Auteur correspondant, E-mail : ovonab@yahoo.fr
}

\section{RESUME}

La détermination des valeurs usuelles des hormones thyroïdiennes et de la typologie biochimique des hyperthyroïdies constituent le but du présent travail. Deux séries de recrutements ont été réalisées à cet effet : 316 sujets pour les valeurs usuelles et 95 hyperthyroïdies des deux sexes appariés pour l'âge (15 à 65 ans). Les valeurs normales des hormones thyroïdiennes rapportées sont (TSHus : 0,13-2,85 mUI/l ; $\mathrm{T}_{3}$ totale : 0,78-2,66 $\mathrm{nmol} / \mathrm{l} ; \mathrm{T}_{4}$ totale : 75,66-116,8 nmol/1 $\left.; \mathrm{FT}_{3}: 2,10-5,84 \mathrm{pmol} / \mathrm{l} ; \mathrm{FT}_{4}: 8,54-14,79 \mathrm{pmol} / \mathrm{l}\right)$. En fonction de cette norme gabonaise, la maladie de Basedow, le goitre secondaire toxique et le goitre multihétéronodulaire toxique sont des hyperthyroïdies s'exprimant au plan biochimique par une élévation de la $\mathrm{FT}_{4}$ et de la $\mathrm{FT}_{3}$, tandis que l'adénome toxique l'est surtout à $\mathrm{FT}_{4}$. Nous soulignons l'intérêt de la détermination des normes de population pour des déductions cliniques et pathogéniques adéquates.

() 2013 International Formulae Group. All rights reserved

Mots clés: Thyroïde, $\mathrm{FT}_{4}, \mathrm{FT}_{3}$, TSH, Normes.

\section{INTRODUCTION}

Les thyréopathies, affections caractérisées par un dysfonctionnement de l'axe hypothalamo-hypophysothyroïdien dans le sens d'une hyperthyroïdie (avec ou sans goitre), ou d'une hypothyroïdie, sont des affections anciennes en Europe et en Asie, mais relativement récentes en Afrique. Une enquête dans une localité du sud-est du Gabon révèle une zone d'endémie goitreuse avec $38 \%$ de cas chez les enfants et $34 \%$ chez les adultes (Ntyonga-Pono, 1993). Autrefois évoquées par la clinique et laborieusement étayées par l'étude du métabolisme de base, du reflexogramme achilléen et de l'iode hormonal, il est possible à l'heure actuelle, grâce à l'application des nouvelles méthodes d'investigation, de pénétrer davantage dans la compréhension du cocktail pathologique de ces affections. En effet, de nombreux travaux ont été réalisés ces derniers temps, de synthèse et de mise au point. Ils ont permis de mettre à la disposition des biologistes, des outils diagnostics simples et fiables et une interprétation appropriée (Watine et al., 2007). La Thyroïd Stimulating Hormon (TSH) ultra sensible et la Free $\mathrm{T}_{4}\left(\mathrm{FT}_{4}\right)$ permettent de nos jours de faire dans $90 \%$ des cas, des 
déductions pathogéniques et cliniques (Wemeau, 1992). Les 10\% des cas restant sont étayés par les anticorps anti récepteurs de la TSH, anti peroxydases, anti récepteurs d'hormones. Par ailleurs, l'application des disciplines d'avenir telles que la génie génétique, permettra d'accéder de façon plus rapide et plus fiable à certaines étiologies d'origine génétique.

Dans nos pays, des difficultés spécifiques existent. D'une part, l'impossibilité de déterminer les anticorps anti-peroxydase, anti-récepteurs TSH, antihormones ainsi que la scintigraphie thyroïdienne, et d'autre part, l'absence de définition et de mise en œuvre des normes (normes individuelles et de population). Toutes ces pesanteurs ne permettent pas toujours une déduction pathogénique et clinique de chaque thyréopathie et ce d'autant que les valeurs de référence de ces hormones sont influencées par la race (Boucai et Surks, 2009). Pour toutes ces raisons, nous nous proposons, dans le présent travail, d'établir la norme de population et de passer en revue les aspects biochimiques des hyperthyroïdies.

\section{MATERIEL ET METHODES \\ Patients}

Deux groupes de sujets ont été utilisés dans ce travail. Le premier était constitué de 316 sujets de tranches d'âge comprises entre 15 et 65 ans dont 210 en enquête déductive (registre des résultats du laboratoire de Biochimie de la Faculté de Médecine de Libreville) et 106 en enquête inductive ont été retenus pour l'établissement des valeurs usuelles des hormones thyroïdiennes dans la population gabonaise (TSH, FT $, \mathrm{FT}_{3}, \mathrm{~T}_{4}$, $\mathrm{T}_{3}$ ).Tous ces sujets avaient un bilan hépatorénal normal et les femmes retenues ne présentaient pas de statut gravidique. De plus, concernant la série des sujets de l'enquête déductive, la méthode de rejet d'éventuels sujets malades décrite par Horn et al. (2001) et déjà exploitée par Rakeda et al. (2009), a été utilisée.

Dans le second groupe, on retrouvait 95 hyperthyroïdiens, bien documentés au plan clinico-biologique et radiologique et devant servir à l'appréciation de l'expression biochimique des hyperthyroïdies au Gabon.

\section{Méthodes}

Les prélèvements de sang veineux périphérique ont été effectués sur tube sec par ponction veineuse au pli du coude entre $8 \mathrm{~h}$ et 8 h30 chez des sujets à jeun depuis 12 heures. Les sérums obtenus par centrifugation à 3000 tours/min pendant 5 minutes, ont été conservés à $-20{ }^{\circ} \mathrm{C}$ jusqu'au moment du dosage.

Les dosages ont été réalisés dans le laboratoire de Biochimie de la Faculté de Médecine. Les tests VIDAS de Biomérieux, ( TSH, $\mathrm{T}_{3}, \mathrm{~T}_{4}, \mathrm{FT}_{3}, \mathrm{FT}_{4}$ ) automatisés sur le système VIDAS et selon les recommandations du fabriquant, permettent la mesure quantitative de la TSH, $\mathrm{T}_{3}$ et $\mathrm{T}_{4}$ totales, $\mathrm{T}_{3}$ et $\mathrm{T}_{4}$ libres dans le sérum ou plasma humain. En résumé, le principe du dosage associait la méthode immuno-enzymatique à une détection finale en fluorescence (ELFA=Enzyme Linked Fluorescent Assay). Toutes les étapes de réactions étaient gérées par l'instrument grâce aux dispositions suivantes: la phase solide et le système de pipetage sont constitués par un cône. Il était sensibilisé par un anticorps monoclonal spécifique de l'hormone à doser (anticorps monoclonal de mouton pour la $\mathrm{T}_{3}$, de lapin pour la $\mathrm{FT}_{4}$ et $\mathrm{T}_{4}$, immunoglobulines monoclonales de souris pour la TSH), une cartouche prête à l'emploi et faite de dix puits gérait le système réactionnel. Le premier puits vide devait recevoir l'échantillon, le contrôle ou le calibrateur, suivi d'autre puits vides pour différentes réactions; puits avec réactifs pré répartis dont le conjugué (phosphatase alcaline), les tampons de lavages successifs. 
Cette cartouche était charriée par un scanner et la détection finale en fluorescence était réalisée dans le dernier puits qui contenait le 4-méthyl-ombelliferyl phosphate. Les coefficients de variation intra et intersérie sont 4,7 et $3,5 \%$ pour la TSH, 6,5 et $8,2 \%$ pour la FT4 et 7,2 et 9,5 pour la FT3 (Erkins, 1990).

La créatinine, les transaminases (ASAT, ALAT) et l'urée ont été dosés dans le sérum par les méthodes classiques chez tous les patients contribuant à l'établissement des valeurs usuelles afin d'éliminer une éventuelle hépathopathie et/ou néphropathie pouvant influencer l'hormonémie.

\section{Analyse statistique}

Les valeurs de référence ont été calculées à l'aide de la méthode décrite par Horn et al. (1998). Les résultat sont été exprimés en médiane avec un intervalle de confiance (IC) à 95\%. La comparaison des médianes a été effectuée à l'aide du test non paramétrique de Kruskal Wallis. Les différences étaient significatives pour des valeurs de $\mathrm{p}<0,05$.

\section{RESULTATS}

Les sujets retenus pour l'établissement des valeurs usuelles, que ce soit en enquête déductive ou inductive, présentaient un bilan hépatorénal normal: alanine aminotransférase et aspartate amino-transférase inférieures à $40 \mathrm{UI} / \mathrm{l}$, l'urée comprise entre 2,5 et $6,6 \mathrm{mmol} / 1$ et des concentrations de créatinine comprises entre 60 et $120 \mu \mathrm{mol} / \mathrm{l}$.

Les résultats obtenus dans la population de référence de notre étude ont été résumés dans le Tableau 1. Ils ont été comparés à ceux d'une population française de la région lyonnaise. Les zones d'euthyroïdies étaient quasiment superposables dans les deux populations en ce qui concerne la $T_{3}$ totale, et plus pincées dans la population gabonaise par rapport à son homologue française pour la $\mathrm{T}_{4}$, la $\mathrm{FT}_{3}$ et la $\mathrm{FT}_{4}$. Ces résultats suggéraient aussi l'existence d'une plus large fourchette de valeurs traduisant une hyperthyroïdie et une zone d'hypothyroïdie plus étroite dans la population gabonaise versus Lyonnaise. On notait également une concordance entre les hormones périphériques et la TSH (Figure 1). La cinétique de production des hormones de l'âge de 15 à plus de 65 ans ne présentait pas de dissociation notable. Les valeurs adultes étaient déjà atteintes entre 15 et 20 ans (Figure 2).

La courbe de distribution de la TSH a été présentée à la Figure 3 . On notait une première partie croissante à partir de 0,2 $\mathrm{mUI} / 1$ pour atteindre un maximum à 0,75 $\mathrm{mUI} / \mathrm{l}$. Cette première phase était suivie d'une seconde correspondant à une décroissance de type exponentielle négative, alors que la distribution des hormones périphériques était quelconque.

L'analyse des profils biochimiques de 95 hyperthyroïdies chez qui le diagnostic étiologique avait été posé, a révélé que la maladie de Basedow (hyperthyroïdie caractérisée par une élévation de la $\mathrm{FT}_{4}$ et de $\mathrm{FT}_{3}$ ) et l'adénome toxique (hyperthyroïdie caractérisée par une élévation de la $\mathrm{FT}_{4}$ seule) s'exprimaient de la même manière vis-à-vis des normes des populations gabonaise et française (Tableau 2).

Pour le goitre secondaire toxique (GIIB) et le goitre multi hétéronodulaire toxique (GMHNT), on retrouvait une augmentation de FT4 et FT3 vis-à-vis de la norme gabonaise. A l'opposé, leur expression biochimique était mixte vis-à-vis de la norme de la population française $(50 \%$ d'hyperthyroïdies caractérisées par une élévation des deux hormones $\mathrm{FT}_{4}$ et $\mathrm{FT}_{3}, 50 \%$ pour la seule $\mathrm{FT}_{4}$ ). 


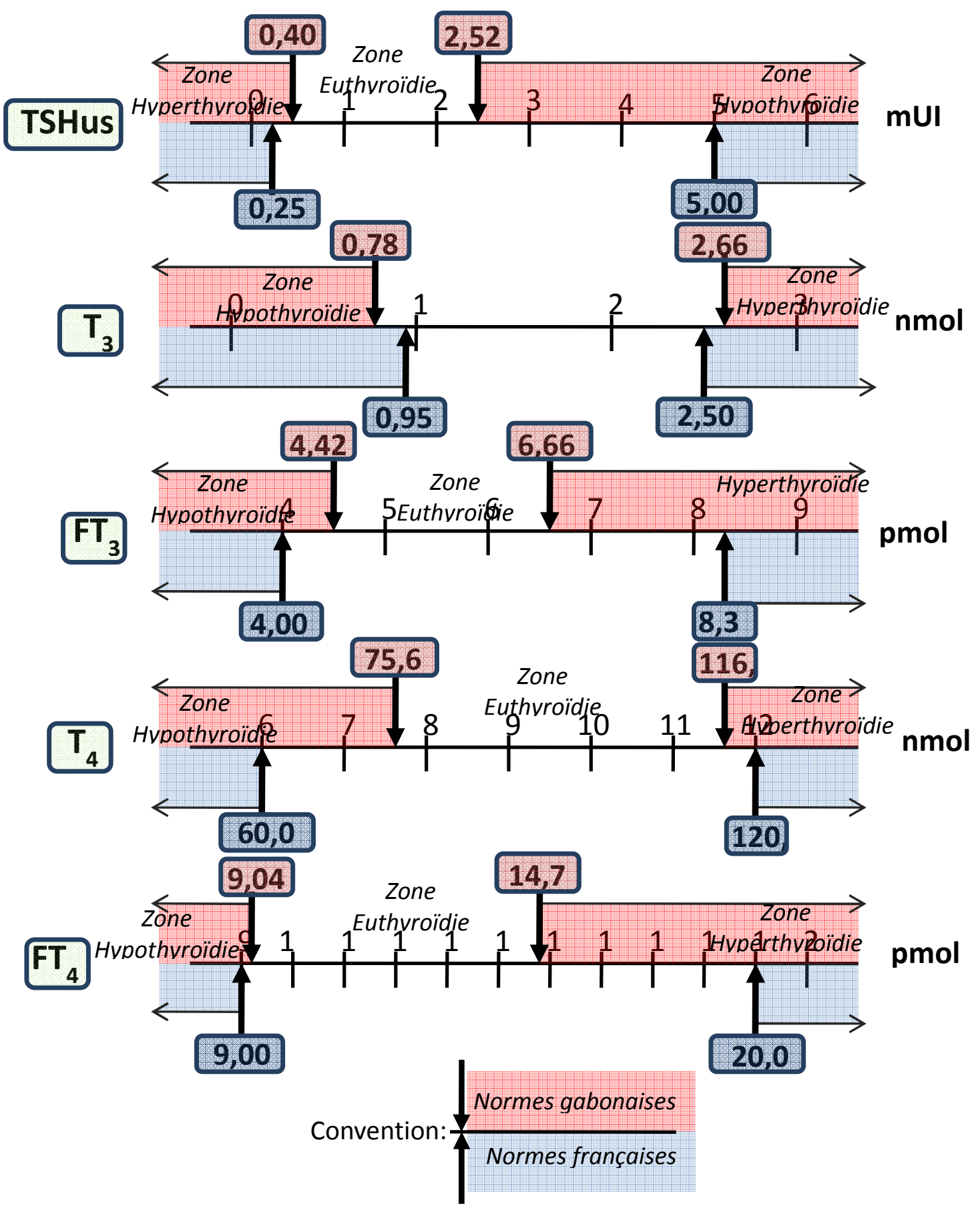

Figure 1: Diagramme des valeurs de référence des hormones. 


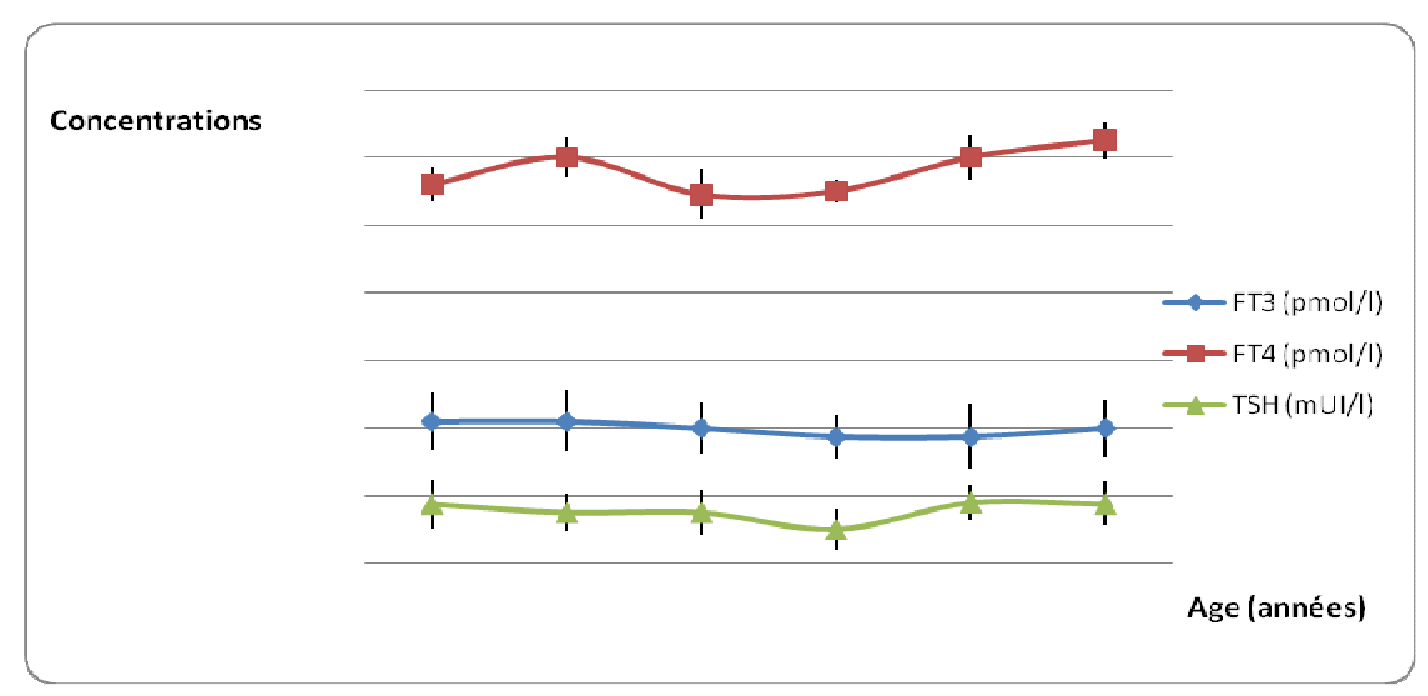

Figure 2: Evolution des hormones thyroïdiennes en fonction de l'âge des sujets témoins.

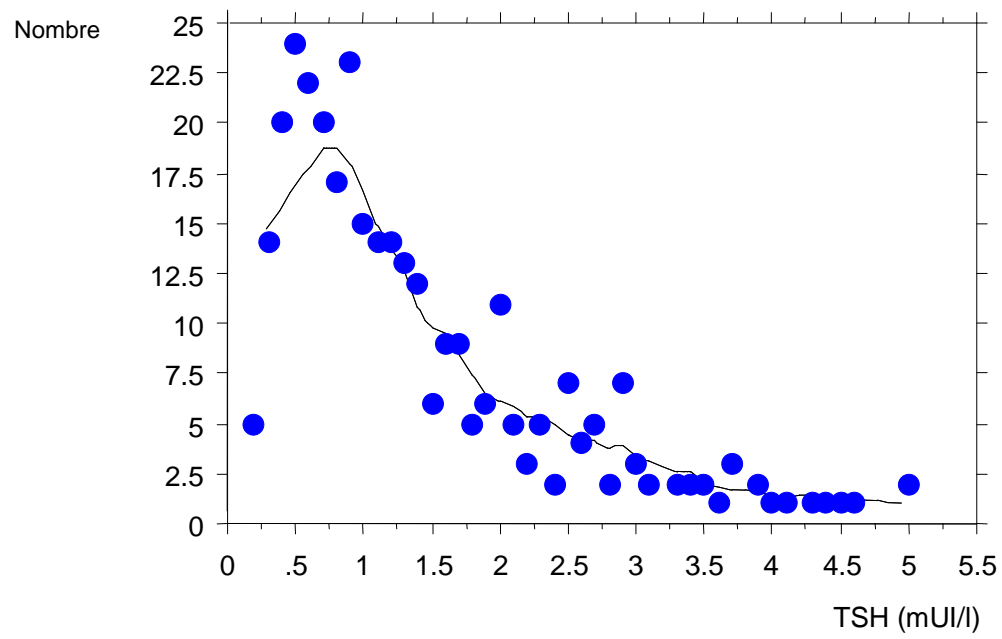

Figure 3: Distribution des valeurs de la TSH chez les sujets témoins. 
Tableau 1 : Comparaison des valeurs usuelles des hormones thyroïdiennes dans les populations gabonaise et européenne (médianes et IC 95\%).

\begin{tabular}{llllll}
\hline \multirow{2}{*}{ Paramètres } & \multicolumn{2}{c}{ Gabon } & \multicolumn{2}{c}{ France } & p \\
\cline { 2 - 6 } & Médianes & IC 95\% & Médianes & IC 95\% \\
\hline TSHus(mUI/l) & 1,49 & $0,13-2,85$ & 2,62 & 0,25 & 0,00 \\
FT4 (pmol/l) & 10,1 & $8,54-11,79$ & 14,5 & $9-$ & 0,00 \\
FT3 (pmol/l) & 3,97 & $2,10-5,84$ & 6,15 & $4-$ & 0,03 \\
T4 (nmol/l) & 96,2 & $75,66-$ & 90 & $60-$ & 0,78 \\
T3 (nmol/l) & 1,72 & $0,78-2,66$ & 1,72 & 0,95 & 0,87 \\
\hline
\end{tabular}

Tableau 2: Expression biochimique des hyperthyroïdies en pourcentage, selon les normes gabonaises et françaises respectivement.

\begin{tabular}{lcccccc}
\hline & \multicolumn{2}{c}{ à $\mathbf{F T}_{\mathbf{3}}$} & \multicolumn{2}{c}{ à $\mathbf{F T}_{\mathbf{4}}$} & \multicolumn{2}{c}{$\mathbf{a ̀ ~} \mathbf{F T}_{\mathbf{3}}$-F $\mathbf{F T}_{\mathbf{4}}$} \\
\cline { 2 - 7 } & $\begin{array}{c}\text { Normes } \\
\text { gabonaises }\end{array}$ & $\begin{array}{c}\text { Normes } \\
\text { françaises }\end{array}$ & $\begin{array}{c}\text { Normes } \\
\text { gabonaises }\end{array}$ & $\begin{array}{c}\text { Normes } \\
\text { françaises }\end{array}$ & $\begin{array}{c}\text { Normes } \\
\text { gabonaises }\end{array}$ & $\begin{array}{c}\text { Normes } \\
\text { françaises }\end{array}$ \\
\hline MB & - & - & $0 \%$ & $95 \%$ & $100 \%$ & $99,05 \%$ \\
GIIB & - & - & $25 \%$ & $50 \%$ & $75 \%$ & $50 \%$ \\
GMHNT & - & - & $0 \%$ & $60 \%$ & $100 \%$ & $40 \%$ \\
AT & - & - & $91,66 \%$ & $91,66 \%$ & $8,34 \%$ & $8,34 \%$ \\
\hline
\end{tabular}

MB : maladie de Basedow ; GIIB : Goitre secondaire toxique ; GMHNT : goitre multihétéronodulaire toxique ;

AT : Adénome toxique

\section{DISCUSSION}

La variabilité biologique, liée aux facteurs environnementaux et génétiques, impose à chaque biologiste une règle essentielle: la détermination des valeurs normales ou usuelles pour chaque population de travail. Dans notre étude, l'évolution des hormones en fonction de l'âge chez les sujets euthyroïdiens mettait en évidence peu de variation dans l'intervalle considéré, confirmant ainsi les résultats de Verburg et al. (2011). Ceci suggère une impossibilité de partitionner les valeurs de ces hormones en fonction de l'âge (Koduah et al., 2004). Par contre, les valeurs de TSH suivaient une distribution classique dénommée Johnson B par Shultzet al. (1985). Cette distribution a été également observée par d'autres auteurs (Zophel et al., 2006 ; Soffiati et al., 2006). De leur côté, la distribution des hormones périphériques était quelconque. Ceci nous a amené à utiliser la méthode non paramétrique d'estimation des intervalles de référence. Très simple à réaliser, bien que n'étant pas aussi précise que l'ensemble des méthodes de transformation des données (Lahti et al., 2004), elle fournit cependant des valeurs acceptables, comme celles qui figurent dans le Tableau 1. Cependant, En Arabie Saoudite, Alqahamatani et al. (2006), ont trouvé des intervalles qui vont de $0,48-6,30 \mathrm{mUI} / \mathrm{l}$ pour la TSH, de 9 à 18,62 pmol/l pour la FT4 et de 
3,39-6,85 pmol/1 pour la FT3. Ces intervalles sont assez différents de ceux que nous avons trouvé. Si la différence entre les hormones peut s'interpréter par les différences dans les modalités de recrutement de la population de travail, il est maintenant reconnu que non seulement les valeurs des hormones thyroïdiennes varient en fonction de la race (Boucail et Surks, 2009), mais également selon le mode d'alimentation des populations concernées (Biassoni et al., 1998). Notre intervalle de référence concernant la TSH est relativement proche de celui proposé par Wartofsky et Dickey (2005), qui est de 0,3 à 2,5 mUI/l. En effet, une fois les contraintes techniques écartées, la principale difficulté dans l'établissement des valeurs de référence de la TSH est son asymétrie de distribution vers les valeurs élevées comme présentés dans nos résultats. Cette asymétrie impose souvent l'utilisation des méthodes non paramétriques ou encore la transformation logarithmique des données initiales. Ces opérations sont responsables d'une erreur d'autant plus importante que les valeurs de TSH sont basses et que l'asymétrie est importante. Une des raisons de cette asymétrie est la présence de maladies thyroïdiennes auto immunes occultes dans la population de référence, sensée être saine (comme la thyroïdite de Hashimoto). De plus, les sujets dont les valeurs de TSH se retrouvent dans cette partie asymétrique ont une prévalence plus élevée d'anticorps antiperoxydase (Wartofsky et Dickey, 2005). Néanmoins, notre intervalle de référence de la TSH nous permet de diagnostiquer de manière plus efficace, les dysthyroïdies asymptomatiques, avant leur expression clinique, dont les hyperthyroïdies subcliniques, avec des valeurs de TSH comprises habituellement entre 0,1 et 0,4 mUI/l (Aghini-Lombardi et al., 1999). Ceci est d'autant plus important que les tests de troisième génération actuellement utilisés peuvent identifier des valeurs de TSH de 0,01 à 0,02 mUI/l (Donangelo et Brauntein, 2011). Malgré le fait que les intervalles de référence individuels soient plus restreints que les intervalles de référence des populations, comme nous le présente Andersen et al. (2002), la TSH varie de façon logarithmique à toute modification de la $\mathrm{T}_{3}$ ou de la $\mathrm{T}_{4}$. Une variation de TSH sérique indique qu'une valeur de $\mathrm{T} 3$ ou de $\mathrm{T}_{4}$ est anormale pour un individu dont ces hormones périphériques se retrouvent dans l'intervalle de référence de la population considérée.

La représentation des valeurs usuelles sous la forme d'un diagramme fait apparaître des fourchettes d'hypo et d'hyperthyroïdie plus larges dans notre population versus population caucasienne. Bieleli (1993) trouve au Congo Démocratique des valeurs d'hormonémie à $300 \mathrm{mmol} / \mathrm{l}$ dans la maladie de Basedow. Lokrou et Koffi (1993) soulignent l'intérêt de relever l'originalité de la maladie de Basedow en milieu tropical. Un autre intérêt que dégage ce mode de présentation est celui de la norme individuelle à différents temps du dosage. Cette notion est importante pour la suspicion et la précocité diagnostique mais aussi pour la prise en charge. Les observations de la littérature rapportent qu'il existe de nombreuses causes d'hyperthyroïdies déterminant chacune un hyperfonctionnement thyroïdien par un mécanisme différent (Wemeau, 1992). Lors de cet hyperfonctionnement, $40 \%$ de la $\mathrm{T}_{3}$ proviennent d'une sécrétion directe de la thyroïde et $60 \%$ de la désiodation de la $\mathrm{T}_{4}$ contre respectivement 20 et $80 \%$ pour un fonctionnement basal. Si l'on considère les normes de la population gabonaise pour l'étude des hyperthyroïdies du Gabonais, la typologie biochimique est conforme à celle de la littérature pour la MB et le GMHNT qui sont à $100 \%$ des hyperthyroïdies à $\mathrm{FT}_{3}-\mathrm{FT}_{4}$ tandis que l'expression biochimique de l'AT vis-à-vis de la norme gabonaise est une hyperthyroïdie à $\mathrm{FT}_{4}$ dans $92,66 \%$ des cas avec en plus dans les $8,34 \%$ des hyperthyroïdies à $\mathrm{FT}_{4}-\mathrm{FT}_{3}$ restants, 33,33\% de syndrome de basse $\mathrm{FT}_{3}$. Wemeau (1992) souligne que l'AT produit une hyperthyroïdie à $\mathrm{T}_{3}-\mathrm{T}_{4}$. Lorsqu'une hyperthyroïdie est à $\mathrm{FT}_{4}$, elle peut être attribuée à la surcharge iodée 
chez le sujet âgé débilité (Wemeau, 1992). Or, notre recrutement est relativement jeune. Il pourrait s'agir d'un problème de désiodation (peut-être un déficit en enzyme désiodase) ou une désiodation impropre produisant une rT3 inactive biologiquement ou alors d'un problème de transfert de la $\mathrm{T}_{3}$ par la globuline fixant la thyroxine (TBG). L'expression du profil biochimique des hyperthyroïdies au Gabon est encore plus hétérogène par rapport à la norme européenne (française) surtout pour les GIIB et les GMHNT. Ces observations montrent l'importance de la norme de la population, mais aussi l'intérêt (pour une meilleure connaissance de la physiopathologie) du dosage des iodures et des thiocyanates dans certaines régions du pays où l'on trouve le plus grand nombre de candidats à la pathologie thyroïdienne. En effet, les gabonais étant dans une zone d'endémicité goitreuse avec déficit en iode, sont particulièrement à risque de développer ces maladies thyroïdiennes auto immunes, comme l'affirment Saranac et al. (2011).

\section{Conclusion}

La typologie clinique des hyperthyroïdies est maintenant bien connue. Cependant, en raison des facteurs environnementaux et génétiques spécifiques, celle biochimique l'est moins en Afrique car liée à la nécessaire détermination des fourchettes de valeurs normales pour chaque population étudiée. Il ressort de notre travail que la MB, le GIIB et le GMHNT sont surtout des hyperthyroïdies à $\mathrm{FT}_{3}-\mathrm{FT}_{4}$ alors que l'AT l'est à $\mathrm{FT}_{4}$. Par ailleurs, 33,33\% des valeurs de $\mathrm{FT}_{3}$ sont basses. L'importance et la spécificité de la norme de la population sont ainsi dégagées mais aussi l'intérêt (pour une meilleure connaissance physiopathologique de cette typologie) du dosage des iodures et des thiocyanates dans certaines régions du pays où l'on rencontre le plus grand nombre de candidat à la pathologie thyroïdienne.

\section{REFERENCES}

Aghini-Lombardi F, Antonangeli L, Martino E, Vitti P, Maccherini D, Leoli F, Rago T, Grasso L, Valeriano R, Balestrieri A, Pinchera A. 1999. The spectrum of thyroid disorders in an iodine-deficient community: the Pescopagano survey. J. Clin. Endocrinol. Metab., 84(2): 561-566.

Alqahamatani M, Tamimi W, Aldaker M, Alenzi F, Tamim H, Alsadhan A. 2006. Young adult reference ranges for thyroid function tests on the Centaur immunoassay analyser. Br. J. Biomed. Sci., 63(4): 163-165.

Andersen S, Pedersen KM, Bruun NH, Laurberg P. 2002. Narrow individual variations in serum T4 and T3 in normal subjects: a clue to the understanding of subclinical thyroid disease. J. Clin. Endocrinol. Metab., 87(3): 1068-1072.

Argemi B. 2000. Exploration biologique de la fonction thyroïdienne. L'indispensable, le confortable, le superflu. Revue de l'ACOMEN, 6(1): 15-21.

Biassoni P, Ravera G, Bertocchi J, Schenone F, Bourdoux P. 1998. Influence of dietary habits on thyroid status of a nomadic people, the Bororo shepherds, roaming a central African region affected by severe iodine deficiency. Eur. J. Endocrinol., 138: $681-685$.

BieleliIsi E. 1993. Le goitre hyperthyroïdien (Issue thérapeutique de 18 cas). Med. Afr. Noire, 40: 527-29.

Boucai L, Surks MI. 2009. Reference limits of serum TSH and free T4 are significantly influenced by race and age in an urban outpatient medical practice. Clin. Endocrinol., 70(5): 788-93.

Delecourt F, Tilly A, Forzy G. 1999. Variation de la TSH (thyroid stimulating hormone) en fonction de l'âge. Ann. Biol. Clin., 57(6): 739-740.

Donangelo I, Braunstein GD. Update on Subclinical Hyperthyroidism. 2011. Am. Fam. Physician, 83(8): 933-938. 
Ekins R. 1990. Measurement of free thyroid hormones in blood. Endocrine Reviews, 11(1): 5-46.

Horn PS, Feng L, Li Y, Pesce AJ. 2001. Effect of outliers and non healthy individuals on reference interval estimation. Clin. Chem., 47(12): 21372145.

Horn PS, Pesce AJ, Copaland BE. 1998. A robust approach to reference interval estimation and evaluation. Clin. Chem., 44(3): 622-631.

Koduah M, Iles TC, Nix BJ. 2004. Centile charts I: new method of assessment for univariate reference intervals. Clin. Chem., 50(5): 901-906.

Lahti A, Petersen PH, Boyd JC, Rustad P, Laake P, Solberg HE. 2004. Partitioning of non-gaussian distributed biochemical reference data into subgroups. Clin. Chem., 50(4): 891-900.

Linnet K. 1987. Two-stage transformation systems for normalization of reference distribution evaluated. Clin. Chem., 33(3): 381-386.

Lokrou A, Koffi JC. 1993. Formes cliniques particulières de la maladie de Basedow en Côte-d'Ivoire. Rev. Franç. Endocrinol. Clin., 34(1): 33-38.

Ntyonga-Pono MP. 1993. Données d'une préenquête sur la prévalence du goitre dans une localité du sud-est du Gabon. Med. Afr. Noire, 40: 543-545.

Rakeda K, Mishiba M, Nakajima A, Kohama M, Hiramatsu S. 2009. Evaluated reference intervals for serum free thyroxine and thyrotropin using conventional outliner rejection test without regard to presence of thyroantibodies and prevalence of thyroid dysfunction in Japanese subjects. Endocrinol. J., 56(9): 1059-1066.

Saranac L, Zivanovic S, Bjelakovic B, Stamenkovic H, Novak M, Kamenov B. 2011. Why is the thyroid so prone to auto immune disease? Horm. Res. Paediatr., 75: 157-165.

Schultz EK, Willard KE, Rich SS, Connelly DP, Critchfield GC.1985. Improved reference interval estimation. Clin. Chem., 31(12): 1974-1978.

Soffiati G, Dirizzi RM, Giavarina D. 2006. Indirect methods for reference intervals based on current data. Clin. Chem., 52(2): 335-336.

Verburg FA, Kirchgassner C, Hebestreit H, Steigerwald U, Lentjes EG, Ergezinger K, Grelle I, Reiners C, Luster M. 2011. Reference ranges for analytes of thyroid function in children. Horm. Metab. Res., 43(6): 422-426.

Wartofsky L, Dickey RA. 2005. Controversy in clinical endocrinology. The evidence for a narrower thyrotropin reference range is compelling. J. Clin. Endocrinol. Metab., 90(9): 5483-5488.

Watine J, Szymanowicz A, Perrin A, Gilbert JC. 2007. Propositions de commentaires interprétatifs pour les bilans biologiques thyroïdiens. Ann. Biol. Clin., 65(3): 317333.

Wemeau JL. 1992. Hyperthyroïdie : Etiologie, Physiopathologie, Diagnostic, Evolution, Pronostic, Principes du traitement. Rev. Prat., 42(7): 857-906.

Zophel K, Wunderlich G, Kotzerke J. 2006. Should we really determine a reference population for the definition of thyroidstimulating hormone reference interval? Clin. Chem., 52(2): 329-330. 